\title{
Maximum antichains in posets of quiver representations
}

\author{
Florian Gellert ${ }^{1}$ - Philipp Lampe P,2 $^{1,2}$
}

Received: 3 April 2017 / Accepted: 12 September 2017 / Published online: 6 October 2017

(C) The Author(s) 2017. This article is an open access publication

\begin{abstract}
We study maximum antichains in two posets related to quiver representations. Firstly, we consider the set of isomorphism classes of indecomposable representations ordered by inclusion. For various orientations of the Dynkin diagram of type A we construct a maximum antichain in the poset. Secondly, we consider the set of subrepresentations of a given quiver representation, again ordered by inclusion. It is a finite set if we restrict to linear representations over finite fields or to representations with values in the category of pointed sets. For particular situations we prove that this poset is Sperner.
\end{abstract}

Keywords Quiver representations · Subrepresentations · Posets · Maximum antichains

Mathematics Subject Classification $16 \mathrm{G} 20 \cdot 06 \mathrm{~A} 07$

Philipp Lampe

lampe@math.uni-bielefeld.de

Florian Gellert

florian.gellert@math.uni-bielefeld.de

1 Faculty of Mathematics, Bielefeld University, PO Box 100 131, 33501 Bielefeld, Germany

2 Department of Mathematical Sciences, Durham University, Science Laboratories, South Road, Durham DH1 3LE, UK 


\section{Introduction and notation}

\subsection{Maximum antichains in posets}

Let $(P, \leq)$ be a poset. Two elements $a, b \in P$ are called incomparable if neither $a \leq b$ nor $b \leq a$ holds. The elements are called comparable otherwise. A subset $\mathscr{F} \subseteq P$ of pairwise incomparable elements is called an antichain. An antichain $\mathscr{F} \subseteq P$ is called maximal if there does not exist an element $a \in P$ such that $\mathscr{F} \cup\{a\}$ is an antichain. It is called maximum if there does not exist an antichain $\mathscr{F}^{\prime} \subseteq P$ such that $\left|\mathscr{F}^{\prime}\right|>|\mathscr{F}|$. Note that every maximum antichain is a maximal antichain, but the converse does not hold in general. The size of a maximum antichain is sometimes called the width of the poset. Furthermore, a subset $C \subseteq P$ of pairwise comparable elements is called a chain. Note that the elements of a chain can be ordered to form a sequence ( $a_{1} \leq a_{2} \leq \cdots \leq a_{k}$ ) and we will often use this notation to describe a chain. Maximal and maximum chains are defined in a similar way to maximal and maximum antichains.

Let $n \geq 1$ be an integer. We denote by $\mathscr{P}_{n}$ the set of all subsets of the finite set $\{1,2, \ldots, n\}$. Note that $\mathscr{P}_{n}$ is partially ordered by inclusion. Sperner (1928) constructs a maximum antichain in $\left(\mathscr{P}_{n}, \subseteq\right)$ :

Theorem 1 (Sperner) The set $\left\{A \in \mathscr{P}_{n}:|A|=\lfloor n / 2\rfloor\right\}$ is a maximum antichain in the poset $\left(\mathscr{P}_{n}, \subseteq\right)$ so that width of the poset is given by $\left(\begin{array}{c}n \\ \lfloor n / 2\rfloor\end{array}\right)$.

Later, Stanley (1991), Theorem 2.2 gives an elegant proof of Sperner's theorem using linear algebra and a grading of the poset. We give a sketch of the proof after recalling some basic notions about posets. We say that the element $a \in P$ covers the element $b \in P$ if $a>b$ and there does not exist an element $c \in P$ with $a>c>b$. Moreover, we say that $a \in P$ is minimal if there does not exist an element $b \in P$ with $b<a$. Dually, we say that $a \in P$ is maximal if there does not exist an element $b \in P$ with $b>a$. The poset $(P, \leq)$ is called graded if there exists a map deg: $P \rightarrow \mathbb{N}$ such that $\operatorname{deg}(a)=0$ for all minimal elements $a \in P$ and $\operatorname{deg}(a)=\operatorname{deg}(b)+1$ whenever $a$ covers $b$. For example, the poset $\mathscr{P}_{n}$ is graded by the cardinality viewed as a map $|\cdot|: \mathscr{P}_{n} \rightarrow \mathbb{N}$. A poset $(P, \leq)$ is called bounded if it contains a miminum, i.e. an element $a \in P$ such that $a \leq b$ for every $b \in P$, and a maximum, i.e. an element $a \in P$ such that $b \leq a$ for every $b \in P$. Note that a finite, bounded poset is graded if and only if all maximal chains have the same cardinality. The rank of a graded poset $(P, \leq)$ is $\operatorname{rk}(P)=\max (\operatorname{deg}(a): a \in P)$.

Suppose that the poset $(P, \leq)$ is indeed finite, bounded and graded of rank $n$. Then we denote by $P_{i} \subseteq P$ the subset of elements of degree $i$. It is easy to see that every $P_{i}$ with $i \geq 0$ is an antichain. We say that $P$ is Sperner if there exists a natural number $i$ such that $|\mathscr{F}| \leq\left|P_{i}\right|$ for every antichain $\mathscr{F} \subseteq P$. In other words, $P_{i}$ is a maximum antichain but there may exist other maximum antichains in $P$. In the case of the power set $\mathscr{P}_{n}$ we simply write $\mathscr{P}_{n, i}$ instead of $\left(\mathscr{P}_{n}\right)_{i}$. Sperner's theorem implies that the power set $\mathscr{P}_{n}$ is Sperner. For every $i$ we consider the $\mathbb{Q}$-vector space $\mathbb{Q} P_{i}$ with basis $P_{i}$. 


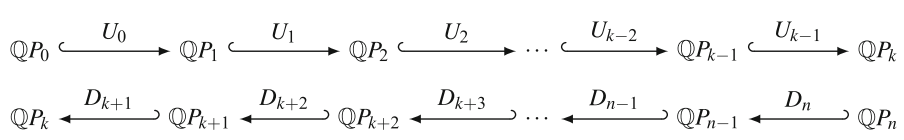

Fig. 1 Injective maps in Stanley's theorem

Theorem 2 (Stanley) Let $k$ be a natural number. Suppose that there are injective linear maps $U_{i}: \mathbb{Q} P_{i} \rightarrow \mathbb{Q} P_{i+1}$ for $0 \leq i \leq k-1$ such that $U_{i}(a) \in \sum_{b \geq a} \mathbb{Q} b$ for every $a \in P_{i}$; suppose there are injective linear maps $D_{i}: \mathbb{Q} P_{i} \rightarrow \mathbb{Q} P_{i-1}$ for $k+1 \leq i \leq n$ such that $D_{i}(a) \in \sum_{b \leq a} \mathbb{Q} b$ for every $a \in P_{i}$, see Fig. 1 for an illustration. Then the poset $(P, \leq)$ is Sperner and $P_{k}$ is a maximum antichain.

As a first application, Stanley gives an elegant proof of Sperner's theorem. A crucial role in the proof is played by the linear maps $U, D: \mathbb{Q} \mathscr{P}_{n} \rightarrow \mathbb{Q} \mathscr{P}_{n}$ defined by the formulae $U(A)=\sum_{b \notin A} A \cup\{b\}$ and $D(A)=\sum_{a \in A} A \backslash\{a\}$ for all $A \in \mathscr{P}_{n}$. Denote by $U_{i}: \mathbb{Q} \mathscr{P}_{n, i} \rightarrow \mathbb{Q} \mathscr{P}_{n, i+1}$ and $D_{i}: \mathbb{Q} \mathscr{P}_{n, i} \rightarrow \mathbb{Q}_{n, i-1}$ the restrictions of $U$ and $D$ to the homogeneous component of degree $i$. An essential observation in Stanley's argument is the commutation relation

$$
D_{i+1} U_{i}-U_{i-1} D_{i}=(n-2 i) \mathrm{id}_{\mathbb{Q}} P_{n, i}
$$

for all $i$. This relation directly implies that $D_{i+1} U_{i}$ is positive definite for all $0 \leq i<\left\lfloor\frac{n}{2}\right\rfloor$. Hence $D_{i+1} U_{i}$ is invertible so that $U_{i}$ must be injective in this case. By a similar argument we can show that $D_{i}$ is injective for $\left\lfloor\frac{n}{2}\right\rfloor<i \leq n$. As a second application, Stanley proves that the poset of vector subspaces of a finite-dimensional vector space over a finite field, again ordered by inclusion, is Sperner.

A chain decomposition of a poset $(P, \leq)$ is a disjoint union

$$
P=C_{1} \sqcup C_{2} \sqcup \cdots \sqcup C_{k}
$$

where every $C_{i}$ is a chain in $P$. If $\mathscr{F} \subseteq P$ is an antichain, then every chain $C_{i}$ contains at most one element of $\mathscr{F}$. Especially, we have $|\mathscr{F}| \leq k$. A chain decomposition is called a Dilworth decomposition if there does not exist a chain decomposition with a smaller number of chains. The next theorem is due to Dilworth (1950), Theorem 1.1:

Theorem 3 (Dilworth) The cardinality of a maximum antichain in a finite poset $(P, \leq)$ is equal to the smallest number of chains in a chain decomposition of $(P, \leq)$.

A chain $C=\left(a_{1} \leq a_{2} \leq \cdots \leq a_{k}\right)$ in $P$ is called saturated if $a_{i+1}$ covers $a_{i}$ for all $1 \leq i \leq k-1$. Suppose that the poset $(P, \leq)$ is graded with degree map $\operatorname{deg}: P \rightarrow \mathbb{N}$ and has finite rank $n=\operatorname{rk}(P)$. A chain $C=\left(a_{1} \leq a_{2} \leq \cdots \leq a_{k}\right)$ in $P$ is called symmetric if it is saturated and the equality $\operatorname{deg}\left(a_{1}\right)+\operatorname{deg}\left(a_{k}\right)=n$ holds. A chain decomposition $P=C_{1} \sqcup C_{2} \sqcup \cdots \sqcup C_{k}$ is called symmetric if every chain $C_{i}$ is symmetric. We say that the graded poset $(P, \leq)$ is a symmetric chain order if it admits a symmetric chain decomposition. Engel (1997), Theorem 5.1.4 proves a relationship between symmetric chain orders and Sperner posets:

Theorem 4 If the graded poset $(P, \leq)$ is a symmetric chain order, then it is Sperner. 
As an application of the theorem, Mühle (2015) shows that certain posets of noncrossing partitions are Sperner. Noncrossing partition posets can be attached to Coxeter groups and play an important role in combinatorics and representation theory.

Suppose that $\left(P, \leq_{P}\right)$ and $\left(Q, \leq_{Q}\right)$ are two posets. The direct sum is the partial order $\leq P \times Q$ on the set $P \times Q$ such that $(a, b) \leq P \times Q \quad(c, d)$ if and only if $a \leq_{P} \quad c$ and $b \leq_{Q} d$. If $P$ and $Q$ are graded posets with degree maps $\operatorname{deg}_{P}$ and $\operatorname{deg}_{Q}$, then the direct product $\left(P \times Q, \leq_{P \times Q}\right)$ is graded with collated degree map $\operatorname{deg}_{P \times Q}(a, b)=\operatorname{deg}_{P}(a)+\operatorname{deg}_{Q}(b)$ for all $a \in P, b \in Q$. The next theorem is a product theorem for symmetric chain orders. The main idea is due to Bruijn et al. (1951) and formal proofs are due to Aigner (1975), Alekseev (1988) and Griggs (1977).

Theorem 5 If the graded posets $\left(P, \leq_{P}\right)$ and $\left(Q, \leq_{Q}\right)$ are both symmetric chain orders, then the direct product $\left(P \times Q, \leq_{P \times Q}\right)$ is a symmetric chain order as well.

Example 1 For a natural number $k$ the poset $C h(k)=(0 \leq 1 \leq \cdots \leq k)$ is called the chain poset of length $k+1$. It becomes a graded poset when we define $\operatorname{deg}(a)=a$ for all $0 \leq a \leq k$. By construction the chain poset $C h(k)$ is a symmetric chain order. For natural numbers $k_{1}, k_{2}, \ldots, k_{r}$ let $C h\left(k_{1}, k_{2}, \ldots, k_{r}\right)$ be the set of all sequences $\left(a_{1}, a_{2}, \ldots, a_{r}\right) \in \mathbb{N}^{r}$ such that $0 \leq a_{i} \leq k_{i}$ for all $1 \leq i \leq r$. We order the set by the direct sum partial order, i.e. we say $\left(a_{1}, a_{2}, \ldots a_{r}\right) \leq\left(b_{1}, b_{2}, \ldots, b_{r}\right)$ if and only if $a_{i} \leq b_{i}$ for all $1 \leq i \leq r$. Clearly, we have $C h\left(k_{1}, k_{2}, \ldots, k_{r}\right)$ $=C h\left(k_{1}\right) \times C h\left(k_{2}\right) \times \cdots \times C h\left(k_{r}\right)$ and the product theorem implies that the poset $C h\left(k_{1}, k_{2}, \ldots, k_{r}\right)$ is a symmetric chain order and hence Sperner. The poset is known as the chain product.

\subsection{Quiver representations}

A quiver $Q=\left(Q_{0}, Q_{1}\right)$ is a finite directed graph with vertex set $Q_{0}$ and arrow set $Q_{1}$. A vertex $i \in Q_{0}$ is called a source if there does not exist an arrow $\alpha \in Q_{1}$ that ends in $i$. Similarly, a vertex $i \in Q_{0}$ is called a sink if there does not exist an arrow $\alpha \in Q_{1}$ that starts in $i$.

We fix a field $k$. A representation of $Q$ is a collection $V=\left(\left(V_{i}\right)_{i \in Q_{0}},\left(V_{\alpha}\right)_{\alpha \in Q_{1}}\right)$ consisting of a finite-dimensional $k$-vector space $V_{i}$ for every vertex $i \in Q_{0}$ and a $k$-linear map $V_{\alpha}: V_{i} \rightarrow V_{j}$ for every arrow $\alpha: i \rightarrow j$ in $Q_{1}$. We denote by $\underline{\operatorname{dim}} V=\left(\operatorname{dim}_{k} V_{i}\right)_{i \in Q_{0}}$ the dimension vector of $V$. The support of $V$ is defined as the set $\operatorname{supp}(V)=\left\{i \in Q_{0}: V_{i} \neq 0\right\}$. Furthermore, the $\operatorname{sum}_{\operatorname{dim}_{k}}(V)=\sum_{i \in Q_{0}} \operatorname{dim}_{k}\left(V_{i}\right)$ is called the dimension of $V$.

A subrepresentation $U$ of $V$ is representation of $Q$ such that $U_{i} \subseteq V_{i}$ is a $k$-vector subspace for every vertex $i \in Q_{i}$ and $U_{\alpha}(x)=V_{\alpha}(x)$ for every arrow $\alpha: i \rightarrow j$ in $Q_{1}$ and every element $x \in U_{i}$. In particular, we have $U_{\alpha}\left(U_{i}\right) \subseteq U_{j}$ for every arrow $\alpha: i \rightarrow j$. Given a subrepresentation $U \subseteq V$, we can define a quotient representation $V / U$ by vector spaces $(V / U)_{i}=V_{i} / U_{i}$ for all vertices $i \in Q_{0}$ and induced canonical maps $(V / U)_{\alpha}: V_{i} / U_{i} \rightarrow V_{j} / U_{j}$ for all arrows $\alpha: i \rightarrow j$. The representation $V$ with $V_{i}=0$ for all $i \in Q_{0}$ is called the zero representation, where necessarily $V_{\alpha}=0$ for all $\alpha \in Q_{1}$. A representation $V$ is called simple if it does not admit a non-zero proper subrepresentation $0 \varsubsetneqq U \varsubsetneqq V$. 
Fig. 2 A path with $n$ vertices

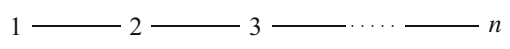

Suppose that $V, W$ are two representations of the same quiver $Q$. A morphism $\phi: V \rightarrow W$ is a collection of $k$-linear maps $\phi_{i}: V_{i} \rightarrow W_{i}$ for all vertices $i \in Q_{0}$ such that $W_{\alpha} \circ \phi_{i}=\phi_{j} \circ V_{\alpha}$ for all arrows $\alpha: i \rightarrow j$ in $Q_{1}$. Representations of $Q$ over $k$ together with their morphisms form a category denoted $\operatorname{rep}_{k}(Q)$. The morphism with $\phi_{i}=0$ for all $i \in Q_{0}$ is called the zero morphism. It is easy to see that a morphism $\phi=\left(\phi_{i}\right)_{i \in Q_{0}}$ is a categorical monomorphism if and only if every linear map $\phi_{i}$ is injective. If $U$ is a subrepresentation of $V$, then the family of canonical inclusions $U_{i} \hookrightarrow V_{i}$ provides a basic example of a monomorphism $\phi: U \rightarrow V$. Dually, a morphism $\phi=\left(\phi_{i}\right)_{i \in Q_{0}}$ is a categorical epimorphism if and only if every linear map $\phi_{i}$ is surjective. It is easy to see that a morphism $\phi=\left(\phi_{i}\right)_{i \in Q_{0}}$ admits an inverse morphism if and only if every linear map $\phi_{i}$ is invertible. Hence a morphism $\phi: V \rightarrow W$ is a categorical isomorphism if and only if it is both a monomorphism and an epimorphism. In this case we say that $V$ and $W$ are isomorphic and we write $V \cong W$.

The category $\operatorname{rep}_{k}(Q)$ is equivalent to the category of finite-dimensional modules over the path algebra $k Q$.

Suppose that $V, W$ are two representations of the same quiver $Q$. The direct sum $V \oplus W$ is the representation with $(V \oplus W)_{i}=V_{i} \oplus W_{i}$ for all vertices $i \in Q_{0}$ and

$$
(V \oplus W)_{\alpha}=\left(\begin{array}{cc}
V_{\alpha} & 0 \\
0 & W_{\alpha}
\end{array}\right): V_{i} \oplus W_{i} \rightarrow V_{j} \oplus W_{j}
$$

for all arrows $\alpha: i \rightarrow j$. A representation is called decomposable if it is isomorphic to a direct sum $V \oplus W$ with $V, W \neq 0$. It is called indecomposable otherwise. Note that every simple representation is indecomposable but the converse statement does not hold in general. A quiver is called representation finite if there are only finitely many indecomposable representations up to isomorphism. It is called representation infinite otherwise.

Let a path of length $n$ be an undirected graph as in Fig. 2. To unify the description of quivers which are representation finite, let us introduce star-shaped undirected graphs as graphs with a central vertex $c$ from which $r$ paths of varying lengths start. More formally, for integers $r \geq 0$ and $\ell_{1}, \ldots, \ell_{r} \geq 1$ let $\operatorname{Star}\left(\ell_{1}, \ldots, \ell_{r}\right)$ be the graph with $n=1+\sum_{i=1}^{r} \ell_{i}$ vertices and edges $c-v_{i, 1}, v_{i, j}-v_{i, j+1}$ for $1 \leq i \leq r$ and $1 \leq j \leq \ell_{i}-1$. Pictorially such a graph can be seen in Fig. 3 .

A star-shaped undirected graph with $n$ vertices is said to be a Dynkin diagram of type $A_{n}$ if $r=1, \ell_{1}=n-1$, it is Dynkin diagram of type $D_{n}$ if $r=3$, $\ell_{1}=\ell_{2}=1, \ell_{3}=n-3$ and Dynkin diagram of type $E_{n}$ if $r=3, \ell_{1}=1, \ell_{2}=2$ and $\ell_{3}=n-4$ for $n \in\{6,7,8\}$.

Gabriel (1972) then classifies representation finite quivers as follows:

Theorem 6 (Gabriel) A (non-empty) connected quiver with $n$ vertices is representation finite if and only if its underlying undirected graph is a Dynkin diagram of type $A_{n}, D_{n}$ or $E_{n}$. In this case, the map $V \mapsto \underline{\operatorname{dim} V}$ induces a bijection between the 


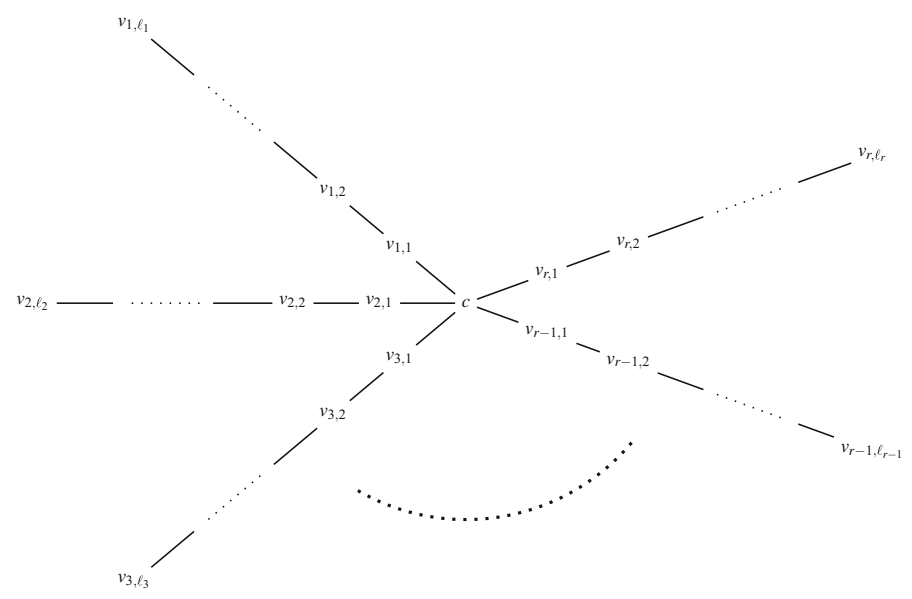

Fig. 3 Star-shaped undirected graph

Fig. 4 Morphisms between indecomposable representations of a quiver of type $A_{2}$
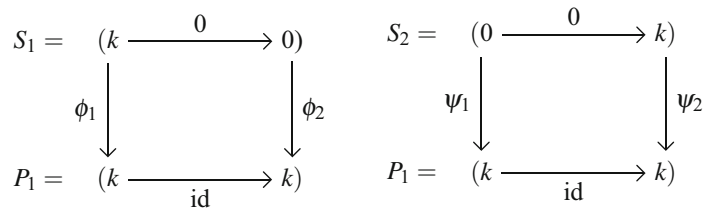

isomorphism classes of indecomposable representations and the positive roots in the corresponding root system.

Especially, representation finiteness only depends on the underlying diagram and not on the orientation. We say that quivers as in Theorem 6 are of type $A_{n}, D_{n}$ and $E_{n}$ respectively and call them Dynkin if we do not wish to distinguish between these three families. For those readers not familiar with representation theory, let us consider one basic example to clarify the notions above.

Example 2 Let $Q$ be the quiver $1 \stackrel{\alpha}{\longrightarrow} 2$ of type $A_{2}$. One representation is then given by $V_{1}=k, V_{2}=0$ and the zero map; denote this representation by $S_{1}=(k \rightarrow 0)$. Since its only proper subrepresentation is the zero representation, we clearly see that it is simple. Similarly, $S_{2}=(0 \rightarrow k)$ is a simple and thus also an indecomposable representation. Gabriel's theorem asserts the existence of a third indecomposable representation with dimension vector $(1,1)$, namely the representation $P_{1}=(k \stackrel{\text { id }}{\longrightarrow} k)$. It is an easy observation that the zero morphism is the only morphism from $S_{1}$ to $P_{1}$, i.e. the left diagram in Fig. 4 commutes if and only if $\phi_{1}=0=\phi_{2}$.

On the other hand, the choice $\psi_{1}=0$ and $\psi_{2}=$ id makes the right diagram of Fig. 4 commutative, hence we obtain a nonzero morphism from $S_{2}$ to $P_{1}$. Since the identity map is injective, the morphism $\left(\psi_{1}, \psi_{2}\right)$ is even a monomorphism of representations. By Gabriel's theorem, a general representation has the form $V=S_{1}^{a} \oplus P_{1}^{b} \oplus S_{2}^{c}$ for some integers $a, b, c \geq 0$, so that $V_{1}=k^{a} \oplus k^{b}, V_{2}=k^{b} \oplus k^{c}$, and $V_{\alpha}=\left(\begin{array}{cc}0 & \text { id } \\ 0 & 0\end{array}\right)$ in block form. 


\section{Maximum antichains in monomorphism posets of indecomposable representations for type $A$ quivers}

In this section, we wish to study maximum antichains in posets attached to various quiver representations.

Gabriel's Theorem 6 provides a nice description of the indecomposable representations of a Dynkin quiver $Q$, which serve as building blocks for all representations of $Q$. Of equal importance are the morphisms between the representations. The morphisms are encoded by the Auslander-Reiten quiver of $Q$ and every arrow in the AuslanderReiten quiver epitomizes either a monomorphism or an epimorpism. Some authors restrict themselves to monomorphisms. The idea goes back to Ringel and Schmidmeier (2008) who introduced monomorphism categories in order to solve a problem of Birkhoff [monomorphism categories are in itself an intense object of study, see the introduction in Zhang (2011) for a short overview of this topic]. In this section we wish to investigate the poset $\left(\mathscr{P}_{Q}, \leq\right)$ by taking indecomposable representations of a Dynkin quiver $Q$ of type $A$ and order them by inclusion.

This poset has a number of interesting properties. For example, a theorem of Oppermann et al. (2015) and Gabriel (1972) asserts that downward closed subsets of $\mathscr{P}_{Q}$ stand in bijection with the elements of the corresponding Weyl group (i.e. the symmetric group $S_{n}$ if $Q$ is any orientation of the Dynkin diagram $A_{n}$ ). Such a downward closed subset is uniquely determined by its maximal elements. The maximal elements form an antichain; conversely every antichain determines a downward closed subset. It is therefore interesting to study large antichains, because they yield large subrepresentation closed categories of representations.

On a related note, in recent work of Ringel (2013), antichains within the root poset of quivers of Dynkin type have been studied when the partial ordering is given by $x \leq y$ if $y-x$ is a non-negative linear combination of elements in the basis of simple roots. For such posets, the maximal cardinality of any antichain clearly equals the number of vertices of the Dynkin diagram in question and antichains of cardinality $n-1$ are considered instead. Rather than following the above approach by studying the dimension vectors of indecomposable representations, for us it seems more natural to investigate the poset of inclusions directly.

\subsection{Poset properties}

Let $n \geq 1$ be a natural number and $Q$ be a Dynkin qiver of type $A_{n}$. For natural numbers $1 \leq a \leq b \leq n$ use the shorthand notation $[a, b]$ for the representation with vector spaces $V_{i}=k$ for $a \leq i \leq b$ and $V_{i}=0$ elsewhere, and linear maps $V_{\alpha}=\left(V_{i} \rightarrow V_{j}\right)=\mathrm{id}_{k}$ for arrows $\alpha: i \rightarrow j$ with $a \leq i, j \leq b$ and $V_{\alpha}=0$ for all others. In the case where $a=b$ we simply write $[a]$ instead of $[a, a]$.

Example 3 Consider again the quiver $Q$ from Example 2. Then the indecomposable modules are $S_{1}=[1], S_{2}=[2]$ and $P_{1}=[1,2]$.

As a consequence of Gabriel's theorem, every indecomposable representation of $Q$ is isomorphic to $[a, b]$ for appropriate choices of $1 \leq a \leq b \leq n$. 

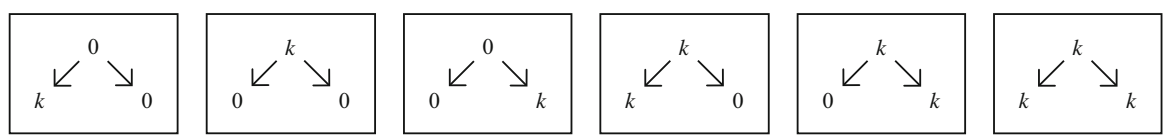

Fig. 5 Indecomposable representations of alternating $A_{3}$

Fig. 6 Hasse diagram of indecomposable representations of alternating $A_{3}$

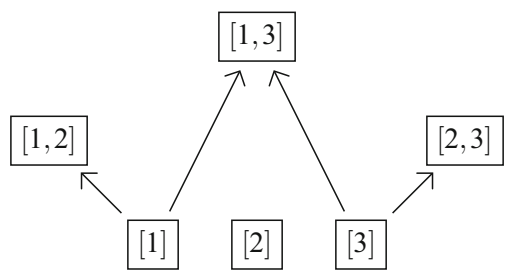

Example 4 Let $Q$ be the quiver $1 \leftarrow 2 \rightarrow 3$. The set of isomorphism classes of indecomposable representations contains 6 elements: [1], [2], [3], [1, 2], [2, 3], [1, 3]. These representations are visualized in Fig. 5. To simplify the notation, whenever we draw an arrow between one-dimensional vector spaces, we assume that the associated map is the identity.

For the rest of this section, let $\left(\mathscr{P}_{Q}, \leq\right)$ be the poset with set

$$
\mathscr{P}_{Q}=\{[a, b]: 1 \leq a \leq b \leq n\}
$$

and $[a, b] \leq\left[a^{\prime}, b^{\prime}\right]$ whenever there exists a monomorphism $[a, b] \hookrightarrow\left[a^{\prime}, b^{\prime}\right]$. Equivalently, we have $[a, b] \leq\left[a^{\prime}, b^{\prime}\right]$ if and only if $[a, b]$ is a subrepresentation of $\left[a^{\prime}, b^{\prime}\right]$.

Example 5 Consider again the quiver as in Example 4. Then $[1] \leq[1,2]$ and $[1] \leq[1,3]$, but $[1,2] \not 1,3]$. The Hasse diagram of this poset is shown in Fig. 6 .

Remark 1 The poset $\left(\mathscr{P}_{Q}, \leq\right)$ for $Q$ a Dynkin quiver of type $A_{n}$ may be graded as in Example 5, but this is not generally the case as we observe later on in Example 6. What is more, for $n>1$ these posets are never bounded and thus never Sperner as the simple representations supported on a source yield isolated vertices in the poset.

\subsection{Linear orientation}

In this and the following subsections, we will restrict to particular orientations of the path from Fig. 2. For now, we consider the linear orientation with unique sink at 1 and unique source at $n$, see Fig. 7 .

Then $[a, b] \leq\left[a^{\prime}, b^{\prime}\right]$ if and only if $a=a^{\prime}$ and $b \leq b^{\prime}$. Hence the poset $\left(\mathscr{P}_{Q}, \leq\right)$ decomposes into $n$ disjoint chains

$$
C_{i}=([i] \leq[i, i+1] \leq[i, i+2] \leq \cdots \leq[i, n])
$$

Fig. 7 Linear orientation of type $A_{n}$

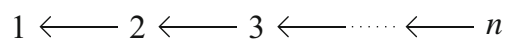




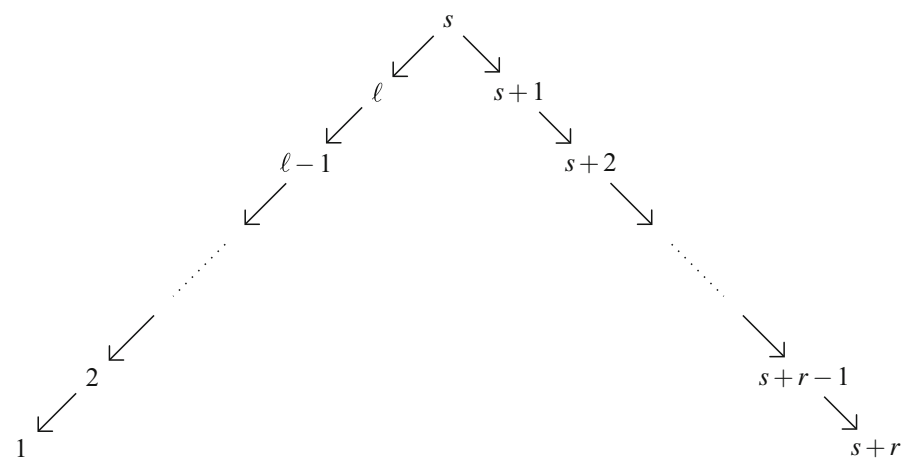

Fig. 8 Simple zigzag orientation of type $A_{n}$

for all $1 \leq i \leq n$ and we immediately obtain the following result.

Proposition 1 A maximum antichain of $\left(\mathscr{P}_{Q}, \leq\right)$ for linearly oriented $A_{n}$ consists of exactly $n$ elements.

\subsection{Simple zigzag}

Let $1 \leq s \leq n$ and consider the orientation of the path of length $n$ with a unique source at position $s$. For $s=1$ or $s=n$ the case degenerates to the linear orientation (up to reordering the vertices) of Sect. 2.2. To simplify the notation, denote $\ell=s-1$ and $r=n-s$ so that the quiver is of the form as shown in Fig. 8.

Theorem 7 The set $\mathscr{F}=\{[a, b]: a \leq s \leq b\}$ is a maximum antichain of size $(\ell+1)(r+1)$ in $\left(\mathscr{P}_{Q}, \leq\right)$.

Proof Let $[a, b]$ and $\left[a^{\prime}, b^{\prime}\right]$ be distinct elements from the set $\mathscr{F}$ and suppose there exists a monomorphism $\phi:[a, b] \hookrightarrow\left[a^{\prime}, b^{\prime}\right]$. This implies $a^{\prime} \leq a$ and $b \leq b^{\prime}$. Since these elements are distinct, $a^{\prime}<a$ or $b<b^{\prime}$. Without loss of generality, we may restrict to $a^{\prime}<a$. Then the linear map corresponding to $\alpha: a \rightarrow(a-1)$ is zero in $[a, b]$ whereas in $\left[a^{\prime}, b^{\prime}\right]$ it is the identity. $\operatorname{But~id}_{k} \circ \phi_{a} \neq \phi_{a-1} \circ 0$ yields a contradiction. Hence $\mathscr{F}$ is an antichain.

To show that $\mathscr{F}$ is a maximum antichain, we describe a particular chain decomposition of $\left(\mathscr{P}_{Q}, \leq\right)$ and observe that $\mathscr{F}$ contains precisely one element of each chain.

For $1 \leq i \leq \ell$ and $1 \leq j \leq r$ let

$$
\begin{aligned}
L_{i} & =([i] \leq[i, i+1] \leq \cdots \leq[i, s]) \\
R_{j} & =([s+j] \leq[s+j-1, s+j] \leq \cdots \leq[s, s+j])
\end{aligned}
$$




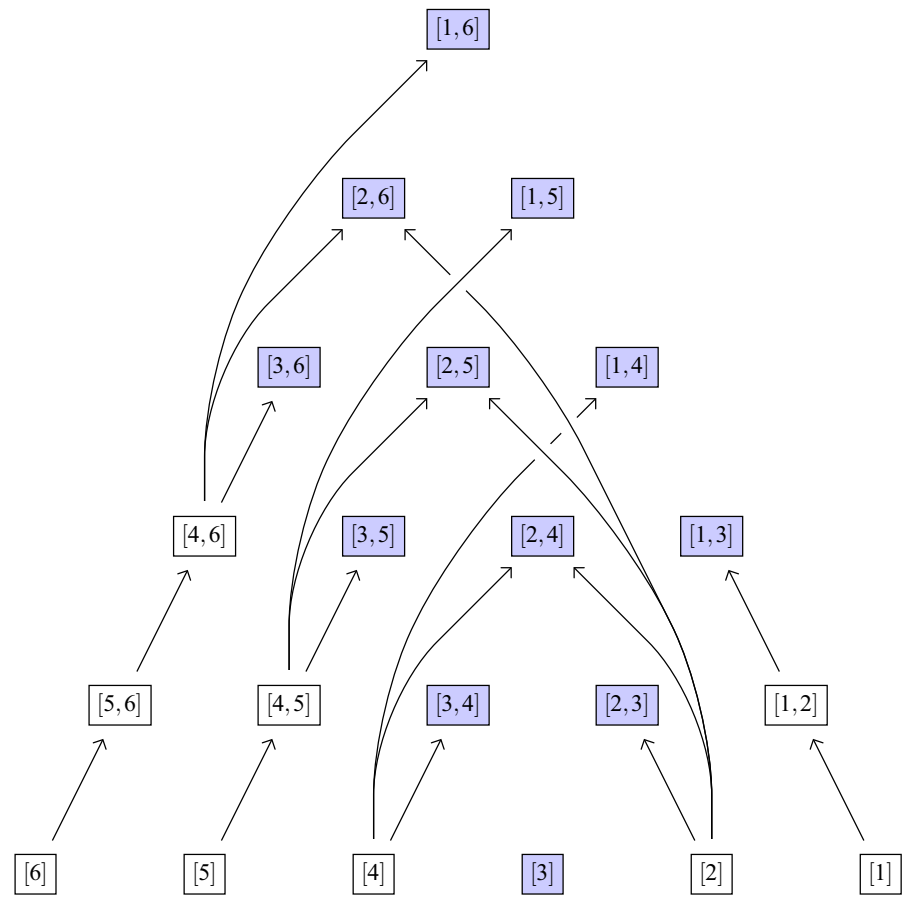

Fig. 9 Hasse diagram of the monomorphism poset of a quiver of type $A_{6}$

We then obtain a chain decomposition of the entire poset $\mathscr{P}$ as follows:

$$
\mathscr{P}=\left(\bigsqcup_{1 \leq i \leq \ell} L_{i}\right) \sqcup\left(\bigsqcup_{1 \leq j \leq r} R_{j}\right) \sqcup\left(\bigsqcup_{a<s<b}\{[a, b]\}\right) \sqcup\{[s]\} .
$$

Note that the sets of indecomposable representations on the right hand side of the union in (1) are to be understood as chains of size one. Every element of $\mathscr{F}$ lies in exactly one chain in (1) as the maximal element. The number of elements in an antichain is thus bounded above by the number of chains in the decomposition in (1), which coincides with the cardinality of $\mathscr{F}$ by construction. This implies that the decomposition in (1) is a Dilworth decomposition and that $\mathscr{F}$ is a maximum antichain.

Example 6 Let $n=6$ and $s=3$, hence $\ell=2$ and $r=3$. The Hasse diagram drawn horizontally and $[a, b] \leq\left[a^{\prime}, b^{\prime}\right]$ indicated by arrows $[a, b] \rightarrow\left[a^{\prime}, b^{\prime}\right]$ is shown in Fig. 9. The elements of the set $\mathscr{F}$ are highlighted.

\subsection{Alternating orientation}

For this subsection, let $m \geq 1$ be a natural number and $n=2 m+1$. Then we consider the quiver $Q$ which arises from the alternating orientation of the path of length $n$ starting and ending with a sink, see Fig. 10. 
Fig. 10 Alternating orientation of path of length $n$ with $m$ sources

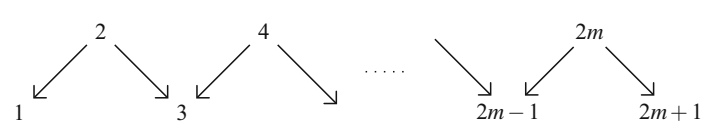

Table 1 Chains depending on a choice of one or two sources

\begin{tabular}{llll}
\hline Chain & & Condition & Cardinality \\
\hline Name & Description & & \\
\hline$C_{[a]}$ & $([a])$ & & 1 \\
$C_{[a, n]}$ & $([a, n])$ & $a \neq 2$ & 1 \\
$C_{[1, a]}$ & $([a-1, a] \leq[a-3, a] \leq \cdots \leq[3, a] \leq[1, a])$ & & $a / 2$ \\
$C_{[a, b]}$ & $([a+1, b-1] \leq[a, b-1] \leq[a, b])$ & $a<b$ & 3 \\
\hline
\end{tabular}

Theorem 8 Let $\mathscr{F}=\mathscr{F}_{s r c} \sqcup \mathscr{F}_{1-s r c} \sqcup \mathscr{F}_{s r c-n} \sqcup \mathscr{F}_{1-n}$ be the union of the following four sets:

$$
\begin{aligned}
\mathscr{F}_{s r c} & =\{[a, b]: a \leq b \text { sources }\}, & \mathscr{F}_{s r c-n} & =\{[a, n]: \text { a source }\}, \\
\mathscr{F}_{1-\text { src }} & =\{[1, b]: \text { b source }\}, & \mathscr{F}_{1-n} & =\{[1, n]\} .
\end{aligned}
$$

Then $\mathscr{F}$ is a maximum antichain of size $\frac{1}{2} m(m+1)+2 m+1=\left(\begin{array}{c}m+2 \\ 2\end{array}\right)+m$.

Proof Using the same arguments as in the proof of Theorem 7, we see that $\mathscr{F}$ is an antichain. And as before, we provide a chain decomposition of the entire poset to show that it is also maximum.

Let $a$ and $b$ be sources. Then consider the chains in Table 1 depending on a choice of one or two sources.

Furthermore, we also consider two chains $C_{[1, n]}$ and $C_{[2, n]}$ not depending on a choice of sources, both of cardinality $m+1$ :

$$
\begin{aligned}
& C_{[1, n]}=([1] \leq[1,3] \leq[1,5] \leq \cdots \leq[1, n]), \\
& C_{[2, n]}=([n] \leq[n-2, n] \leq[n-4, n] \leq \cdots \leq[5, n] \leq[3, n] \leq[2, n]) .
\end{aligned}
$$

By construction, all of the chains above are pairwise disjoint. Altogether these chains exhaust all elements of the poset since

$$
\begin{aligned}
\frac{n(n+1)}{2} & =\frac{(2 m+1)(2 m+2)}{2}=2 m^{2}+3 m+1 \\
& =m \cdot 1+(m-1) \cdot 1+\frac{m(m+1)}{2}+\left(\begin{array}{c}
m \\
2
\end{array}\right) \cdot 3+2 \cdot(m+1) .
\end{aligned}
$$

Thus they form a chain decomposition of $\left(\mathscr{P}_{Q}, \leq\right)$ and every element of $\mathscr{F}$ lies in exactly one chain as the maximal element. 


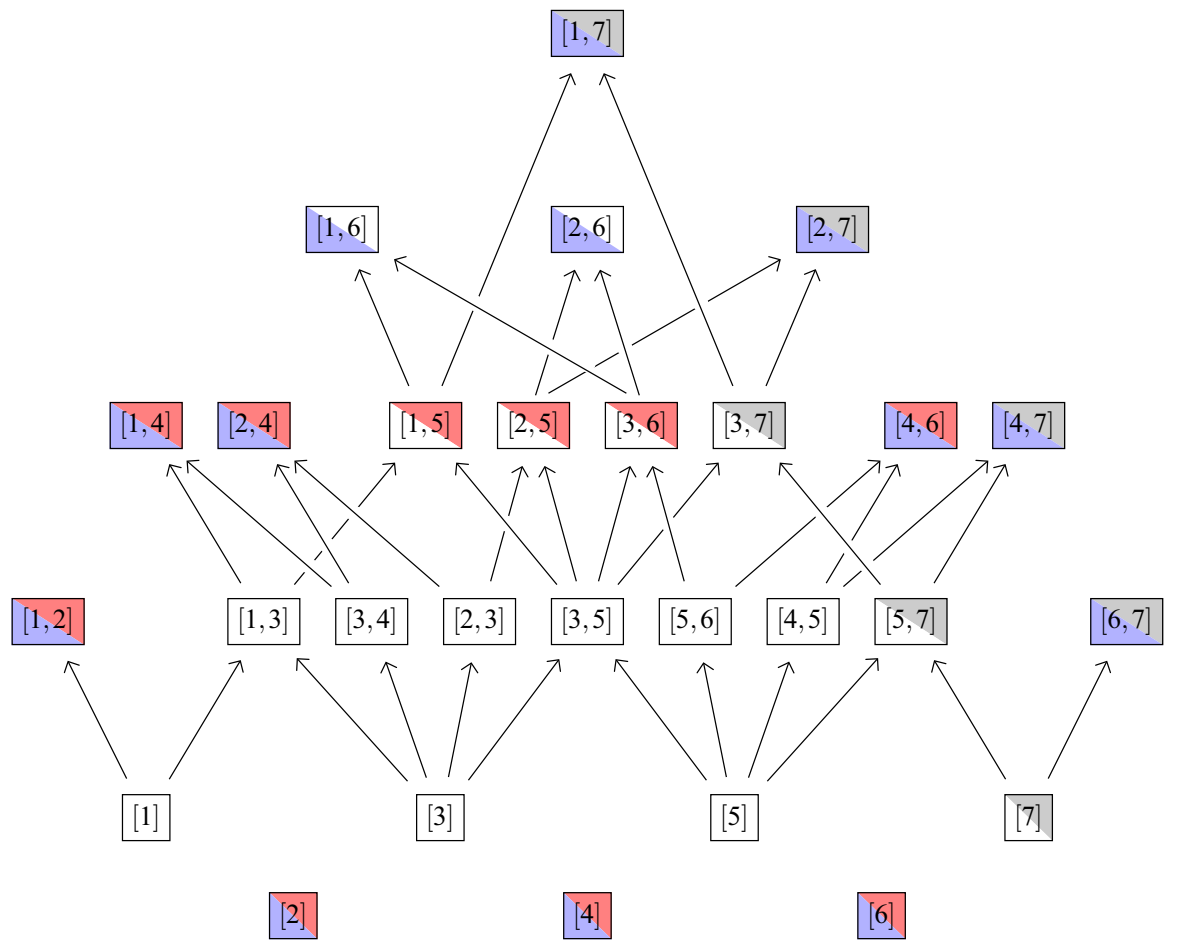

Fig. 11 Hasse diagrams of alternating orientations of $A_{6}$ and $A_{7}$

We can apply this construction to a particular subquiver of $Q$. Let $Q^{\prime}$ be the full subquiver of $Q$ with the vertex $n$ deleted, i.e. the alternating orientation of the path of length $n-1$ starting with a sink and ending with a source. Then we notate $n^{\prime}=n-1$ and observe that $Q^{\prime}$ has indeed $n^{\prime}=2 m$ vertices and $m$ sources.

Corollary 1 Let $\mathscr{F}_{s r c}^{\prime}=\mathscr{F}_{s r c} \backslash\left\{\left[2, n^{\prime}\right]\right\}$ and $\mathscr{F}_{1-s r c}^{\prime}=\mathscr{F}_{1-s r c} \backslash\left\{\left[1, n^{\prime}\right]\right\}$. Then

$$
\mathscr{F}^{\prime}=\mathscr{F}_{s r c}^{\prime} \sqcup \mathscr{F}_{1-s r c}^{\prime} \sqcup\left\{\left[1, n^{\prime}-1\right]\right\} \sqcup\left\{\left[2, n^{\prime}-1\right]\right\} \sqcup\left\{\left[3, n^{\prime}\right]\right\}
$$

is a maximum antichain of $\left(\mathscr{P}_{Q^{\prime}}, \leq\right)$ of cardinality $\frac{1}{2}(m+1)(m+2)$.

Proof The Dilworth decomposition in the proof of Theorem 8 degenerates to a Dilworth decomposition of $\mathscr{P}_{Q^{\prime}}$ if one removes all those elements supported at vertex $n$.

Example 7 Let us consider the case for $m=3$, hence $n=7$ and $n^{\prime}=6$. The Hasse diagrams of the posets $\left(\mathscr{P}_{Q}, \leq\right)$ and $\left(\mathscr{P}_{Q^{\prime}}, \leq\right)$ are shown in Fig. 11. Those nodes contained in $\mathscr{P}_{Q}$ but not in $\mathscr{P}_{Q^{\prime}}$ are shaded gray above the downward diagonal. The elements in $\mathscr{F}$ are highlighted in blue below the downward diagonal and those of $\mathscr{F}^{\prime}$ in red above the downward diagonal. 
Remark 2 For the only case where the alternating orientation of this section coincides with the simple zigzag of Sect. 2.3, the maximum antichains of Theorems 7 and 8 are identical.

Other orientations of the diagram $A_{n}$ than the ones discussed above are less symmetric and thus allow for higher combinatorial complexity. The constructions of Dilworth decompositions do not generalise to arbitrary orientations and computational experiments suggest that the combinatorics of those cases are much more intricate than what we have previously seen. Nevertheless, it should be feasible to obtain similar results with the same techniques as above for particular orientations of quivers of type $D_{n}$. In addition, it would be interesting to see for which orientation of the diagram $A_{n}$ the poset $(\mathscr{P}, \leq)$ has largest width, and whether this maximum is obtained by a simple zigzag with the unique source in the middle of the quiver.

\section{Sperner theorems for subrepresentation posets in type $A$}

\subsection{A Sperner theorem for subrepresentations posets in type $A_{2}$}

Let $V$ be a representation of a quiver $Q=\left(Q_{0}, Q_{1}\right)$ over a field $k$. We denote by $\mathscr{P}_{V}$ the set of all subrepresentations $U \subseteq V$. Assume that $U_{1}, U_{2} \in \mathscr{P}_{V}$. We say that $U_{1} \leq U_{2}$ if and only if $U_{1}$ is a subrepresentation of $U_{2}$. In this way, $\left(\mathscr{P}_{V}, \leq\right)$ becomes a partially ordered set.

Proposition 2 If every simple representation of $Q$ is 1-dimensional, then the dimension function $U \mapsto \operatorname{dim}_{k}(U)$ defines a grading of the subrepresentation poset $\left(\mathscr{P}_{V}, \leq\right)$.

Proof The only minimal element in $\mathscr{P}_{V}$ is the zero representation which satisfies $\operatorname{dim}(0)=0$. Suppose that $U_{2} \in \mathscr{P}_{V}$ covers $U_{1} \in \mathscr{P}_{V}$, i.e. $U_{1} \leq U_{2}$ and there does not exist an element $U_{3} \in \mathscr{P}_{V}$ such that $U_{1}<U_{3}<U_{2}$. The third isomorphism theorem implies that the quotient representation $U_{2} / U_{1}$ is nonzero and that there does not exist a representation $0 \varsubsetneqq U \varsubsetneqq U_{2} / U_{1}$. Thus $U_{2} / U_{1}$ is simple and the assumption implies $\operatorname{dim}_{k}\left(U_{2}\right)-\operatorname{dim}_{k}\left(U_{1}\right)=1$.

Note that the condition of the previous proposition is satisfied if the quiver $Q$ does not contain oriented cycles which we assume from now on. Additionally, we assume that $k=\mathbb{F}_{q}$ is a finite field $\mathbb{F}_{q}$ with $q$ elements. For every natural number $n \in \mathbb{N}$ we define the Gaussian integer as $[n]_{q}=\left(q^{n}-1\right) /(q-1)$. Note that $[n]_{q}=1+q+q^{2}$ $+\cdots+q^{n-1}$ can be simplified to a polynomial in $q$ which specializes to $n$ when we plug in $q=1$. Moreover, $[n]_{q}$ is equal to the number of 1 -dimensional vector subspaces of $\mathbb{F}_{q}^{n}$. The polynomial $[n]_{q} !=[1]_{q} \cdot[2]_{2} \cdots[n]_{q}$ is called Gaussian factorial. For a natural number $0 \leq d \leq n$ the polynomial $\left(\begin{array}{l}n \\ d\end{array}\right)_{q}=[n]_{q} ! /\left([d]_{q} ! \cdot[n-d]_{q} !\right)$ is called Gaussian binomial coefficient. Note that $\left(\begin{array}{l}n \\ d\end{array}\right)_{q}$ is equal to the number of $d$-dimensional vector subspaces of $\mathbb{F}_{q}^{n}$. Likewise the number of $(d+1)$-dimensional $k$-vector spaces $U$ such that $k^{d} \subseteq U \subseteq k^{n}$ is equal to $[n-d]_{q}$. Dually, the number of $(n-1)$-dimensional vector spaces $U$ such that $k^{d} \subseteq U \subseteq k^{n}$ is also equal to $[n-d]_{q}$. 
Theorem 9 If $Q=(1 \rightarrow 2)$ is the quiver of type $A_{2}$ and $V=P_{1}^{a}$ is a direct sum of copies of the indecomposable, projective representation $P_{1}$, then the subrepresentation poset $\left(\mathscr{P}_{V}, \leq\right)$ is Sperner.

Proof Note that the rank of the poset is equal to $\operatorname{dim}_{k}(V)=2 a$. For brevity we write $\mathscr{P}_{i, V}$ instead of $\left(\mathscr{P}_{V}\right)_{i}$ for $0 \leq i \leq 2 a$. A subrepresentation $X \subseteq V$ is given by two vector spaces $0 \subseteq X_{1} \subseteq X_{2} \subseteq k^{a}$. We apply Stanley's Theorem 2. For every integer $i$ we define two maps

$$
\begin{aligned}
U_{i}: \mathbb{Q} \mathscr{P}_{i, V} & \rightarrow \mathscr{P}_{i+1, V}, \quad D_{i}: \mathbb{Q} \mathscr{P}_{i, V} \rightarrow \mathscr{P}_{i-1, V}, \\
X & \mapsto \sum_{\substack{Y \in \mathscr{P}_{i+1, V} \\
Y \geq X}} Y, \quad X \mapsto \sum_{\substack{Z \in \mathscr{P}_{i-1, V} \\
Z \leq X}} Z .
\end{aligned}
$$

for every $X \in \mathscr{P}_{i, V}$ and extend linearly. By construction $D_{i}$ is adjoint to $U_{i-1}$ for every $i$. We can generalize Stanley's commutation relation for the up and down operators from type $A_{1}$ to type $A_{2}$. More precisely, we claim

$$
\left(D_{i+1} \circ U_{i}-U_{i-1} \circ D_{i}\right)(X)=\left(\left[a-d_{2}\right]_{q}-\left[d_{1}\right]_{q}\right) X
$$

for every $X \in \mathscr{P}_{i, V}$ with dimension vector $\left(d_{1}, d_{2}\right)$.

For a proof of the claim, let $X \in \mathscr{P}_{i, V}$. We compute the coefficients of the expansion of $\left(D_{i+1} \circ U_{i}-U_{i-1} \circ D_{i}\right)(X)$ in the standard basis $\mathscr{P}_{i, V}$ of $\mathbb{Q}_{i, V}$. We distinguish the following cases. First, suppose that $X^{\prime} \in \mathscr{P}_{i, V}$ is a representation such that $X_{1}^{\prime} \subseteq X_{1}$ and $X_{2} \subseteq X_{2}^{\prime}$ are both subspaces of codimension 1. In this case, $\left(X_{1} \subseteq X_{2}^{\prime}\right)$ and $\left(X_{1}^{\prime} \subseteq X_{2}\right)$ are both subrepresentations of $V$ so that the coefficient corresponding to $X^{\prime}$ in the expansion of $\left(D_{i+1} \circ U_{i}-U_{i-1} \circ D_{i}\right)(X)$ is equal to $1-1=0$. Second, suppose that $X^{\prime} \in \mathscr{P}_{i, V}$ is a representation such that $X_{1} \subseteq X_{1}^{\prime}$ and $X_{2}^{\prime} \subseteq X_{2}$ are both subspaces of codimension 1 . With the same arguments as before the coefficient corresponding to $X^{\prime}$ in the expansion of $\left(D_{i+1} \circ U_{i}-U_{i-1} \circ D_{i}\right)(X)$ is equal to $1-1=0$. Third, suppose that $X^{\prime} \in \mathscr{P}_{i, V}$ is a representation such that $X_{1}^{\prime}=X_{1}$ and $X_{2}^{\prime} \neq X_{2}$. Then the coefficients corresponding to $X^{\prime}$ in the expansions of $\left(D_{i+1} \circ U_{i}\right)(X)$ and $\left(U_{i-1} \circ D_{i}\right)(X)$ are zero unless $\operatorname{dim}_{k}\left(X_{2} \cap X_{2}^{\prime}\right)=d_{2}-1$ which is equivalent to $\operatorname{dim}_{k}\left(X_{2}+X_{2}^{\prime}\right)=d_{2}+1$. In this case, the coefficient corresponding to $X^{\prime}$ in the expansion of $\left(D_{i+1} \circ U_{i}-U_{i-1} \circ D_{i}\right)(X)$ is equal to $1-1=0$. The fourth case where $X^{\prime} \in \mathscr{P}_{i, V}$ is a representation such that $X_{2}^{\prime}=X_{2}$ and $X_{1}^{\prime} \neq X_{1}$ is treated similarly. Fifth, suppose that $X^{\prime}=X$. The coefficient corresponding to $X^{\prime}$ in the expansion of $\left(D_{i+1} \circ U_{i}\right)(X)$ is equal to the number of $\left(d_{1}+1\right)$-dimensional vector spaces $Y_{1}$ such that $X_{1} \subseteq Y_{1} \subseteq X_{2}$ plus the number of $\left(d_{2}+1\right)$-dimensional vector spaces $Y_{2}$ such that $X_{2} \subseteq Y_{2} \subseteq k^{a}$, namely $\left[d_{2}-d_{1}\right]_{q}+\left[a-d_{2}\right]_{q}$. Moreover, the coefficient corresponding to $X^{\prime}$ in the expansion of $\left(U_{i-1} \circ D_{i}\right)(X)$ is equal to the number of $\left(d_{1}-1\right)$-dimensional vector spaces $Z_{1}$ such that $0 \subseteq Z_{1} \subset X_{1}$ plus the number of $\left(d_{2}-1\right)$-dimensional vector spaces $Z_{2}$ such that $X_{1} \subseteq Z_{2} \subseteq X_{2}$, namely $\left[d_{1}\right]_{q}+\left[d_{2}-d_{1}\right]_{q}$. The difference of the two terms is equal to the term $\left[a-d_{2}\right]_{q}-\left[d_{1}\right]_{q}$ from Eq. (2). For all other cases $X^{\prime}$, the coefficient correspond- 


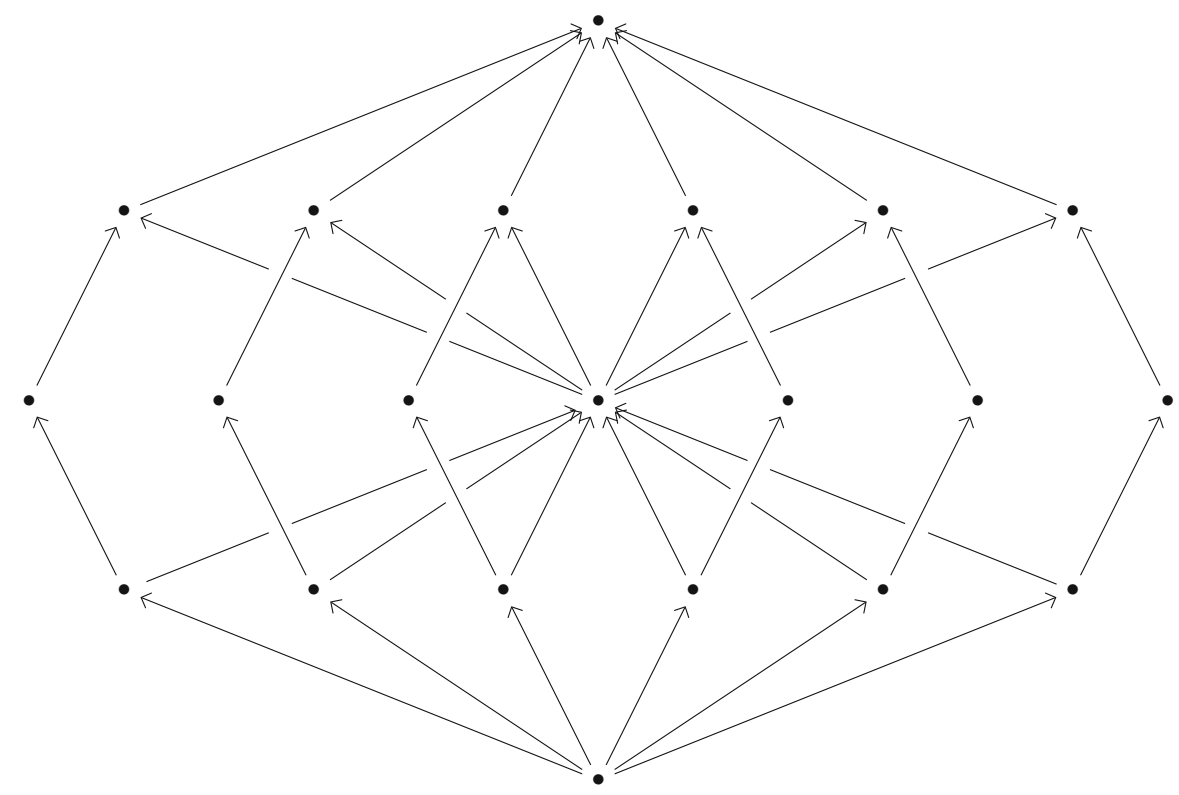

Fig. 12 Subrepresentation poset in type $A_{2}$

ing to $X^{\prime} \in \mathscr{P}_{i, V}$ in the expansion of $\left(D_{i+1} \circ U_{i}-U_{i-1} \circ D_{i}\right)(X)$ is equal to 0 .

Next, we claim that if $0 \leq i<a$, then $U_{i}$ is injective. Dually, if $a<i \leq 2 a$, then $D_{i}$ is injective. We prove the first statement only, the second statement can be proved using the same arguments. The assumption $i<a$ implies $0<a-i=a-d_{2}-d_{1}$ for every $X \in \mathscr{P}_{i, V}$. It follows that $a-d_{2}>d_{1}$ and $\left[a-d_{2}\right]_{q}>\left[d_{1}\right]_{q}$ for every $X \in \mathscr{P}_{i, V}$. By Eq. (2) the matrix of the linear map $D_{i+1} \circ U_{i}-U_{i-1} \circ D_{i}$ (with respect to the standard basis) is a diagonal matrix with positive diagonal entries. Especially, it is positive definite. Moreover, the adjointness of $U_{i-1}$ and $D_{i}$ implies that the matrix corresponding to the composition $U_{i-1} \circ D_{i}$ (with respect to the standard basis) is positive semi-definite. The sum of the two matrices must be positive definite as well. Especially, $D_{i+1} \circ U_{i}$ must be invertible so that $U_{i}$ is injective.

Example 8 Figure 12 shows the poset of subrepresentations of the 4-dimensional representation $P_{1}^{2}$ over the finite field with 5 elements. The subrepresentations of dimension 2 form a maximum antichain of cardinality 7 .

Remark 3 Let $Q=(1 \rightarrow 2 \rightarrow \cdots \rightarrow n)$ for some $n \geq 1$, and let $V=P_{1}^{a}$ for some $a \geq 0$. We believe that the poset $\left(\mathscr{P}_{V}, \leq\right)$ is Sperner in general. In addition to the case in Theorem 9, we were able to verify all choices for $n, a$ and $q$ with $(n, a, q) \in\{3\} \times\{1,2,3\} \times\{2,3\}$ by computer calculations. Unfortunately, the above method of proof does not work when $n \geq 3$ for the following reason. Suppose that $X \subseteq$ $V$ is a subrepresentation with dimension vector $\left(d_{1}, d_{2}, \ldots, d_{n}\right)$. Then the coefficient 
corresponding to $X$ in $\left(D_{i+1} \circ U_{i}\right)(X)$ is equal to $\sum_{r=1}^{n}\left[d_{r+1}-d_{r}\right]_{q}$ whereas the coefficient corresponding to $X$ in $\left(U_{i-1} \circ D_{i}\right)(X)$ is equal to $\sum_{r=0}^{n-1}\left[d_{r+1}-d_{r}\right]_{q}$. Here we interpret $d_{n+1}=a$ and $d_{0}=0$. The difference of the two sums is equal to $\left[a-d_{n}\right]_{q}-\left[d_{1}\right]_{q}$. This term is not positive for all $X$ with $\operatorname{dim}_{k}(X) \leq \frac{1}{2} \operatorname{dim}_{k}(V)$, which would enable us to draw similar conclusions as in the proof of Theorem 9.

\subsection{A Sperner result for subrepresentation posets over pointed sets}

In this section we will study quiver representations with values in the category of pointed sets.

As the authors' background and main interest lies in the study of cluster algebras Fomin and Zelevinsky (2002), Fomin and Zelevinsky (2003), Berenstein et al. (2005) and Fomin and Zelevinsky (2007), let us shortly describe the connection between these algebras and pointed sets. Caldero-Chapoton's prominent cluster character Caldero and Chapoton (2006) relates quiver representations on the one side with cluster algebras on the other. It assigns to a representation $V$ of a Dynkin quiver $Q$ with $n$ vertices a Laurent polynomial $X_{V} \in \mathbb{Z}\left[x_{1}^{ \pm 1}, x_{2}^{ \pm 1}, \ldots, x_{n}^{ \pm 1}\right]$. We may think of it as the multivariate generating function of the Euler characteristics $\chi\left(\mathrm{Gr}_{d}(V)\right)$ of the Grassmannian varieties of subrepresentations $U \subseteq V$ for various dimension vectors $\operatorname{dim}(U)=d$. The Euler characteristics are usually computed by counting subrepresentations over $\mathbb{F}_{q}$ and then specializing $q=1$. Under this specialisation, the counting problem to determine the Euler characteristic of a given representation often becomes a counting problem over the category of pointed sets. So it might be interesting to study the subrepresentation poset of a representation over the category of pointed sets. In light of this connection between cluster algebras, quiver respresentations and pointed sets, studying the latter objects might lead to new insights for and a better understanding of the intricate combinatorics in cluster algebras.

A pointed set $\left(A, 0_{A}\right)$ is a set $A$ together with a distinguished element $0_{A} \in A$. The set is also known as a based set with basepoint $0_{A}$. A pointed subset of a pointed set $\left(A, 0_{A}\right)$ is a subset $B \subseteq A$ that contains $0_{A}$ as its basepoint. Let $\left(A, 0_{A}\right)$ and $\left(B, 0_{B}\right)$ be two pointed sets. A morphism between $\left(A, 0_{A}\right)$ and $\left(B, 0_{B}\right)$ is a map $f: A \rightarrow B$ with $f\left(0_{A}\right)=0_{B}$ such that the restriction $\left.f\right|_{A \backslash f^{-1}\left(O_{B}\right)}: A \backslash f^{-1}\left(O_{B}\right) \rightarrow B$ is injective. The pointed sets together with the morphisms form the category of pointed sets. Some authors also use the term vector spaces over the field with one element. Especially, we say that the morphism $f:\left(A, 0_{A}\right) \rightarrow\left(B, 0_{B}\right)$ is an isomorphism if there exist a morphism $g:\left(B, 0_{B}\right) \rightarrow\left(A, 0_{A}\right)$ such that $g \circ f=\mathrm{id}_{A}$ and $f \circ g=\mathrm{id}_{B}$. Note that in this case $f$ and $g$ are bijections and we say that $\left(A, 0_{A}\right)$ and $\left(B, 0_{B}\right)$ are isomorphic and we write $\left(A, 0_{A}\right) \cong\left(B, 0_{B}\right)$. The direct sum $\left(A, 0_{A}\right) \oplus\left(B, 0_{B}\right)$ of two pointed sets is the set

$$
C=\left(A \backslash\left\{0_{A}\right\}\right) \sqcup\left(B \backslash\left\{0_{B}\right\}\right) \sqcup\left\{0_{C}\right\}
$$

with basepoint $0_{C}$. We have canonical inclusions $\iota_{A}: A \rightarrow C$ and $\iota_{B}: B \rightarrow C$ and canonical projections $\pi_{A}: C \rightarrow A$ and $\pi_{B}: C \rightarrow B$ such that $\pi_{A} \circ \iota_{A}=\mathrm{id}_{A}$ and $\pi_{B} \circ \iota_{B}=\operatorname{id}_{B}$. Moreover, we define the dimension $\operatorname{dim}\left(A, 0_{A}\right)=|A|$ of a pointed 
Fig. 13 A star shaped quiver of type $E_{6}$

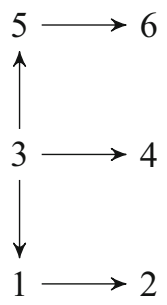

set to be the cardinality of the underlying set. Up to isomorphism there is exactly one pointed set of dimension 0 which we sometimes abbreviate as 0 .

Let $Q=\left(Q_{0}, Q_{1}\right)$ be a quiver. A representation of $Q$ with values in the category of pointed sets is a collection $X=\left(\left(X_{i}, 0_{X_{i}}\right)_{i \in Q_{0}},\left(X_{\alpha}\right)_{\alpha \in Q_{1}}\right)$ consisting of a pointed set $\left(X_{i}, 0_{X_{i}}\right)$ for every vertex $i \in Q_{0}$ and a morphism of pointed sets $X_{\alpha}:\left(X_{i}, 0_{X_{i}}\right) \rightarrow\left(X_{j}, 0_{X_{j}}\right)$ for every arrow $\alpha: i \rightarrow j$ in $Q_{1}$. Szczesny (2012) studies such representations. Let us recall some basic constructions.

First, there is the zero representation with $X_{i}=0$ for all vertices $i \in Q_{0}$. A subrepresentation $U$ of $X$ is representation of $Q$ such that $\left(U_{i}, 0_{U_{i}}\right) \subseteq\left(X_{i}, 0_{X_{i}}\right)$ is a pointed subset for every vertex $i \in Q_{i}$ and $U_{\alpha}(u)=V_{\alpha}(u)$ for every arrow $\alpha: i \rightarrow j$ in $Q_{1}$ and every element $u \in U_{i}$. Especially we have $U_{\alpha}\left(U_{i}\right) \subseteq U_{j}$ for every arrow $\alpha$. A representation is called simple if it does not admit a nonzero proper subrepresentation. Suppose that $X, Y$ are two representations of the same quiver $Q$ with values in the category of pointed sets. A morphism $\phi: X \rightarrow Y$ is a collection of morphisms of pointed sets $\phi_{i}:\left(X_{i}, 0_{X_{i}}\right) \rightarrow\left(Y_{i}, 0_{Y_{i}}\right)$ for all vertices $i \in Q_{0}$ such that $X_{\alpha} \circ \phi_{i}=\phi_{j} \circ Y_{\alpha}$ for all arrows $\alpha: i \rightarrow j$ in $Q_{1}$. It is called an isomorphism if every $X_{\alpha}$ is an isomorphism in the category of pointed sets. In this case we say that $X$ and $Y$ are isomorphic and we write $X \cong Y$.

The direct sum $X \oplus Y$ is the representation with $(X \oplus Y)_{i}=\left(X_{i}, 0_{X_{i}}\right) \oplus\left(Y_{i}, 0_{Y_{i}}\right)$ for all vertices $i \in Q_{0}$ and $(X \oplus Y)_{\alpha}(x)=X_{\alpha}(x)$ and $(X \oplus Y)_{\alpha}(y)=Y_{\alpha}(y)$ for all elements $x \in X_{i} \backslash\left\{0_{X_{i}}\right\}, y \in Y_{i} \backslash\left\{0_{Y_{i}}\right\}$ and all arrows $\alpha: i \rightarrow j$ in $Q_{1}$. A representation is called decomposable if it is isomorphic to a direct sum $X \oplus Y$ with $X, Y \neq 0$. It is called indecomposable otherwise. Szczesny (2012), Theorem 5 describes indecomposable representations with values in the category of pointed sets for quivers whose underlying undirected diagram is a tree. In this case, the isomorphism classes of indecomposable representations correspond to connected subquivers $Q^{\prime}$ of $Q$. The indecomposable representation $X^{\prime}$ corresponding to $Q^{\prime}$ satisfies $\operatorname{dim}\left(X_{i}, 0_{X_{i}}\right)=1$ if $i \in Q^{\prime}$ and $\operatorname{dim}\left(X_{i}, 0_{X_{i}}\right)=0$ otherwise.

Let $X$ be a representation of $Q$ with values in the category of pointed sets. We denote by $\mathscr{P}_{X}$ the finite set of all subrepresentations $U$ of $X$. As before, we define a partial order on the set $\mathscr{P}_{X}$ by putting $U_{1} \leq U_{2}$ if and only if $U_{1}$ is a subrepresentation of $U_{2}$. As in Sect. 3.1 the poset is graded when we define a degree map dim: $\mathscr{P}_{X} \rightarrow \mathbb{N}$ by $\operatorname{dim}(X)=\sum_{i \in Q_{0}} \operatorname{dim}\left(X_{i}\right)$. The following proposition is immediate:

Proposition 3 Let $X$ and $Y$ be two representations of $Q$. Then the poset $\left(\mathscr{P}_{X \oplus Y}, \leq\right)$ is isomorphic to the direct product $\left(\mathscr{P}_{X}, \leq\right) \times\left(\mathscr{P}_{Y}, \leq\right)$. 


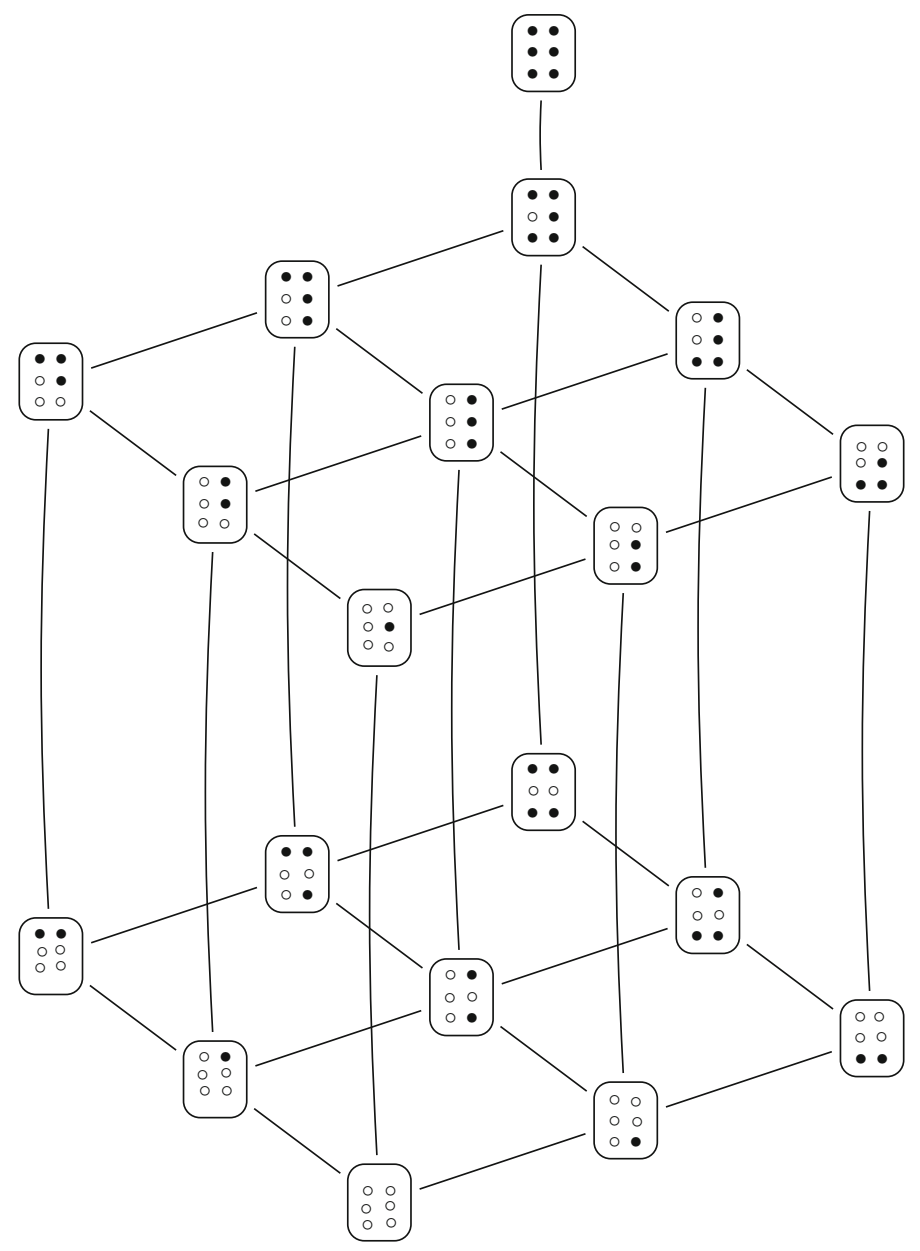

Fig. 14 The Hasse diagram of a subrepresentations poset over pointed sets

Proposition 4 Let $r \geq 1$ and $\ell_{1}, \ell_{2}, \ldots, \ell_{r} \geq 1$ be integers. We consider the quiver $Q$ with underlying undirected star-shaped graph as in Sect. 1.2, with a unique source $c$ and $r$ rays

$$
c \rightarrow v_{1, i} \rightarrow v_{2, i} \rightarrow \cdots \rightarrow v_{i, \ell_{i}}
$$

for $1 \leq i \leq r$. Let $X$ be an indecomposable representation of $Q$ with values in the category of pointed sets. Then $\left(\mathscr{P}_{X}, \leq\right)$ is Sperner.

Proof Without loss of generality we may assume $\operatorname{supp}(X)=Q_{0}$. The case $r=1$ is easy so let us assume $r \geq 2$. A maximum antichain cannot contain the representation $X$ itself. Now notice that $\left(\mathscr{P}_{X} \backslash\{X\}, \leq\right)$ is isomorphic to the chain product $C h\left(\ell_{1}, \ell_{2}, \ldots, \ell_{r}\right)$ from Example 1. The desired result follows. 
A similar statement is true for the star-shaped quiver whose vertices are oriented towards the inner vertex.

Example 9 Let us illustrate the Proposition 4 for $r=3$ and $\left(l_{1}, l_{2}, l_{3}\right)=(2,1,2)$. In this case $Q$ is the Dynkin quiver of type $E_{6}$ in Fig. 13. According to Szcesny's theorem every indecomposable representation $X$ satisfies $\operatorname{dim}\left(X_{i}, 0_{x_{i}}\right) \in\{0,1\}$ for all $i \in Q_{0}$. We denote the 0 -dimensional pointed set by $\circ$ and the 1 -dimensional pointed set by $\bullet$. Now let $X$ be the indecomposable representation with $X_{i}=\bullet$ for all $i \in Q_{0}$ and $X_{\alpha}=\mathrm{id}$. for all $\alpha \in Q_{1}$. A subrepresentation $Y \subseteq X$ satisfies $Y_{i} \in\{\circ, \bullet\}$ for all $i \in Q_{0}$, and a map $Y_{\alpha}: Y_{i} \rightarrow Y_{j}$ is necessarily the identity map if $Y_{i}=Y_{j}=\bullet$. The Hasse diagram of the poset $\left(\mathscr{P}_{X}, \leq\right)$ is shown in Fig. 14. Note that the subrepresentations of dimension 3 form a maximum antichain of size 5 .

Open Access This article is distributed under the terms of the Creative Commons Attribution 4.0 International License (http://creativecommons.org/licenses/by/4.0/), which permits unrestricted use, distribution, and reproduction in any medium, provided you give appropriate credit to the original author(s) and the source, provide a link to the Creative Commons license, and indicate if changes were made.

\section{References}

Aigner, M.: Symmetrische Zerlegungen von Kettenprodukten. Monatshefte Mathematik 79(3), 177-189 (1975)

Alekseev, V.B.: Use of symmetry in finding the width a partially ordered set. Problemy Kibernetiki 28, 5-24 (1988)

Berenstein, A., Fomin, S., Zelevinsky, A.: Cluster algebras. III. Upper bounds and double Bruhat cells. Duke Math. J. 126(1), 1-52 (2005)

de Bruijn, N.G., van Ebbenhorst Tengbergen, C.A., Kruyswijk, D.: On the set of divisors of a number. Nieuw Archief voor Wiskunde 23(2), 191-193 (1951)

Caldero, P., Chapoton, F.: Cluster algebras as Hall algebras of quiver representations. Commentarii Mathematici Helvetici 81(3), 595-616 (2006)

Dilworth, R.: A decomposition theorem for partially ordered sets. Ann. Math. 51(1), 161-166 (1950)

Engel, K.: Sperner Theory. Cambridge University, Cambridge (1997)

Fomin, S., Zelevinsky, A.: Cluster algebras. I. Foundations. J. Am. Math. Soc. 15(2), 497-529 (2002)

Fomin, S., Zelevinsky, A.: Cluster algebras. II. Finite type classification. Invent. Math. 154(1), 63-121 (2003)

Fomin, S., Zelevinsky, A.: Cluster algebras. IV. Coefficients. Compos. Math. 143(1), 112-164 (2007)

Gabriel, P.: Unzerlegbare Darstellungen I. Manuscripta Mathematica 6, 71-103 (1972)

Gottwald, A.: Cofinite submodule closed categories and the Weyl group. Preprint arXiv:1705.04280 (2017)

Griggs, J.R.: Symmetric chain orders, Sperner theorems, and loop matchings. PhD thesis, Massachusetts Institute of Technology (1977)

Mühle, H.: Symmetric chain decompositions and the strong Sperner property for noncrossing partition lattices. Preprint arXiv:1509.06942 (2015)

Oppermann, S., Reiten, I., Thomas, H.: Quotient closed subcategories of quiver representations. Compos. Math. 151, 568-602 (2015)

Ringel, C.M.: The (n-1)-antichains in a root poset of width n. Preprint arXiv:1306.1593 (2013)

Ringel, C.M., Schmidmeier, M.: The Auslander-Reiten translation in submodule categories. Trans. Am. Math. Soc. 360, 691-716 (2008)

Szczesny, M.: Representations of Quivers over $\mathbb{F}_{1}$ and Hall algebras. Int. Math. Res. Notices 2012(10), 2377-2404 (2012)

Sperner, E.: Ein Satz über Untermengen einer endlichen Menge. Mathematische Zeitschrift 27(1), 544-548 (1928)

Stanley, R.: Some applications of algebra to combinatorics. Discrete Appl. Math. 34, 241-277 (1991) 
Zhang, P.: Monomorphism categories, cotilting theory, and Gorenstein-projective modules. J. Algebra 339(1), 181-202 (2011) 